\title{
Lens Designs for High Irradiance Application of Multi-Kilowatt Nd:YAG Welding Lasers
}

\author{
D. M. Keicher and M. Essien \\ Sandia National Laboratories \\ Albuquerque, New Mexico 87185-1411
}

\begin{abstract}
$\underline{\text { Abstract }}$
Multi-kilowatt Nd:YAG lasers provide an appealing solution for aluminum laser welding applications due to increased bulk absorption and ease of beam delivery as compared to high power $\mathrm{CO}_{2}$ laser systems. However, high numerical aperture optics are required to overcome the relatively poor beam quality associated with these lasers and to achieve a high irradiance. Several lens designs have been developed and evaluated to achieve the high irradiance values required to provide good coupling into aluminum alloys. The results of these tests demonstrate that near diffraction limited performance can be achieve for high numerical aperture elements. Additionally, an inverse-telephoto lens design has been developed and characterized to further demonstrate the feasibility of producing a high irradiance with a functional working distance from the weld surface.
\end{abstract}

\section{Introduction}

The recent development of multi-kilowatt $\mathrm{cw} \mathrm{Nd:YAG} \mathrm{lasers} \mathrm{provides} \mathrm{an} \mathrm{alternative} \mathrm{laser}$ source for applications previously dominated by $\mathrm{CO}_{2}$ lasers. The Nd:YAG laser has several advantages over the $\mathrm{CO}_{2}$ laser which have provided an impetus for its development. One advantage is supplied by the absorption characteristic of many metals. In general, the bulk absorption in metals decreases with increasing wavelength[1]. For high conductivity metals such as aluminum, this effect is particularly pronounced in comparing bulk absorption properties at the $\mathrm{CO}_{2}$ and the much shorter $\mathrm{Nd}$ :YAG laser wavelength. It is not uncommon for the $\mathrm{CO}_{2}$ laser to decouple completely from workpiece during processing, thus inhibiting welding. A second, and possibly the most significant advantage offered by the Nd:YAG laser over the $\mathrm{CO}_{2}$ laser is in the ease of beam delivery. The transmission of $\mathrm{Nd}: \mathrm{YAG}$ radiation through tens or hundreds of meters of fiber optic cable is possible with little loss in beam power[2]. This capability makes the $\mathrm{Nd}$ :YAG laser particularly appealing for robotics applications and situations requiring either beam sharing or remote working locations. Finally, since the focused beam spot size is proportional to the square root of the wavelength ${ }^{[3]}$, the $\mathrm{Nd}: Y A G$ laser should ultimately provide a higher irradiance.

However, there are also characteristics provided by the current state of the art Nd:YAG laser that can inhibit its usefulness. The most significant limitation is in the beam quality available from these laser sources. For one commercial Nd:YAG laser system, the beam quality has been reported to be as high as 99 times diffraction limited ${ }^{[4]}$ as compared to reported values of 1.5 times 


\section{DISCLAMER}

Portions of this document may be illegible in electronic image products. Images are produced from the best available original document. 
diffraction limited for a fast axial flow $\mathrm{CO}_{2}$ laser ${ }^{[5]}$ at comparable power levels. This value for the $\mathrm{Nd}$ :YAG laser is extremely poor. Since the beam quality defines the focusability of the laser beam and the amount of spreading which will occur for a laser beam propagating through space, the poor beam quality provided by the Nd:YAG laser may severely limit its performance. The larger angular spreading necessitates the use of larger diameter optics for a direct delivery system located relatively close to the laser source. Since the spot size is limited by the beam quality, it is often necessary to resort to short focal length, high numerical aperture optics to achieve a high irradiance.

However, as the objective lens focal length is decreased, other problems can occur. Since the working distance of the lens decreases with decreasing focal length, in the harsh environment provided by a welding laser, vapor and particles of the molten metal become a problem. Furthermore, recent characterization results for commercially available beam focusing optics suggest that a shorter focal length lens may not provide a reduction in spot size that is inversely proportionate to the lens focal length. Indeed, it has been shown that spherical aberration effects can lead to a larger focused beam spot size with a shorter focal length lens ${ }^{[6]}$.

In understanding these problems, a series of optical elements have been developed that allowed a high irradiance to be obtained from the Nd:YAG laser, in spite of the beam quality problems. This laser system characterized and then, using these results, high numerical aperture (small f/\# lenses) aspheric and multi-element lenses were developed to overcome spherical aberration effects. Experimental results show a reduced spot size for both corrected elements. To overcome the short working distance provided by these small $\mathrm{f} / \#$ lenses, the design sequence was taken a step further to obtain a lens with a similar f/\# with a working distance of approximately two times that of the original multi-element lens design.

\section{Spherical Aberration}

Before discussing the lens design, it is important to understand aberration effects for an optical source. In this particular situation, transmissive optics are used and oriented such that the lens optical axis is parallel to the beam propagation direction. Furthermore, the lens and beam optical axes are coincident. Thus, only spherical aberration need be considered for a monochromatic source such as a laser. Other aberrations such as coma, astigmatism, etcetera only occur for an off-axis source[7].

Ray-tracing equations describing the propagation of a ray of light through any optical system provide an exact solution for image formation. The relationship for the paraxial image formation equation for an optically thin element in air is given as

$$
\frac{1}{f}=\frac{1}{S}+\frac{1}{S^{\prime}}
$$


where $f$ is the lens focal length, $S$ is the object distance from the lens, and $S^{\prime}$ is the image distance from the lens. This relationship, as well as other paraxial imaging equations, provides a good approximation for imaging in cases where the lens focal length is large as compared to lens aperture. This relationship assumes that all rays make small angles with the lens optical axis such that Snell's law of refraction at an optical boundary can be approximated as

$$
n_{1} \theta_{1}=n_{2} \theta_{2}
$$

where $n_{1}$ and $n_{2}$ are the refractive index of the incident and refracting media, and $\theta_{1}$ and $\theta_{2}$ are the angle of incidence and angle of refraction, respectively. As the focal length of the optical element becomes a significant fraction of the lens aperture, this approximation is no longer valid.

For a group of rays propagated through an optical system to form an image, each of the rays, regardless of its direction, must have an identical optical path length. It is the difference in optical path length which gives rise to aberration effects. To understand spherical aberration*, consider the laser beam to consist of a packet of rays propagating through space at slightly different angles which form a spherical wave front incident on a single refracting surface. This is depicted in Fig. 1 (a). For the rays to focus to the paraxial focus position $P_{o}$, then a spherical wave front given as $\sigma$ must exist after refraction. However, if the refracting surface $\mathrm{R}_{\mathrm{S}}$ is spherical and has a radius of curvature located at $O$, upon refraction, the beam wave front ( $\sigma^{\prime}$ in Fig. 1 (b)) will not remain spherical. This is due to an increase in the optical path length of the paraxial rays as compared to the marginal rays (i.e. rays far from the optical axis or making a large angle with optical axis). Since the new surface curvature $\sigma^{\prime}$ is not spherical, then the rays located at different positions from the optical axis will cross the optical axis at different distances from the refracting surface. The rays close to the optical axis will focus at the paraxial focus position while the marginal rays will focus to a position $\mathrm{P}^{\prime}$ closer to the lens. The difference in optical path length of the ideal reference surface $\sigma$ and the real surface $\sigma^{\prime}$ is the origin for spherical aberration. As shown in Fig. 1(b), this effect becomes more pronounced as the beam divergence angle is increased. The effect is also a function of the lens radius of curvature and refractive index. As the lens radius of curvature decreases, the spherical aberration effect increases.

\section{Design Approach}

The laser used for this design was an $1800 \mathrm{~W} \mathrm{cw} \mathrm{Nd:YAG} \mathrm{laser.} \mathrm{Prior} \mathrm{to} \mathrm{designing} \mathrm{the}$ laser lens sets, it was necessary to measure the output beam characteristics of our laser. The beam parameter measurement technique used in characterization of this laser has been described previously[4]. The measured values for beam quality ranged from 58 to 99 times diffraction limited at low and high powers, respectively. Of particular importance in the lens design was the beam divergence angle $\theta$. This angle contains the spherical wave front information to be used in defining boundary conditions for the lens design. As previously shown, the difference between

\footnotetext{
${ }^{*}$ For a more detailed description of spherical aberration, the reader is referred to reference 7.
} 
the refracted laser wave front and the ideal reference sphere determines the difference in optical path length between the paraxial and marginal rays.

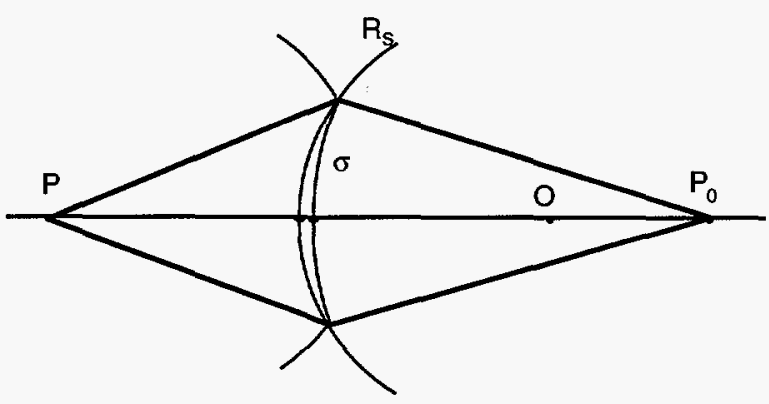

(a)

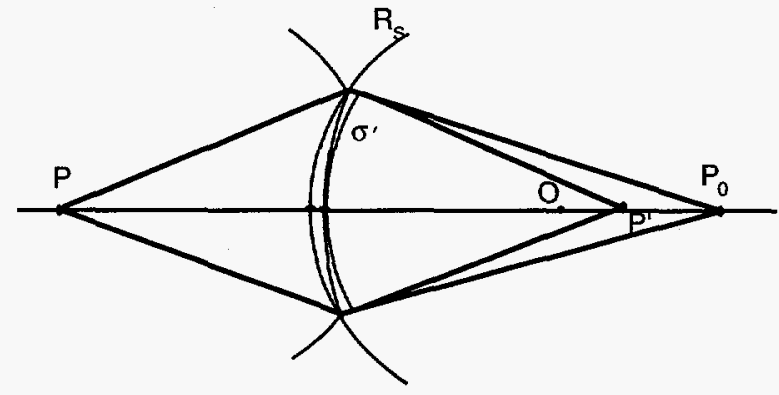

(b)

Figure 1. Schematic representation showing (a) ideal reference sphere required for focusing to point $\mathrm{P}_{0}$, (b) non spherical wave front produced upon refraction from a spherical surface.

The spherical aberration effect can be overcome using several techniques. A relatively simple design, with a complicated fabrication approach, is to use aspheric surfaces to produce a spherical wave front upon refraction. This will allow the beam to be focused to a minimum spot size as allowed by the laser beam quality. However, for the high power Nd:YAG laser used in our work, fused silica had to be used for all of optical elements. This necessitated hand grinding and polishing techniques for fabrication of aspheric elements. This is a time consuming process and each of the elements is unique. Furthermore, to stay within a reasonable cost, compromises in optical quality must be made. However, there is an advantage provided by the single element approach in maximizing the lens working distance.

A second technique for reducing aberration is to use a multi-element approach with all spherical optics. The total aberration of a lens system is the sum of the individual aberration contributions due to each of the optical surfaces ${ }^{[7]}$. The aberration contribution can have both 
positive and negative values. By proper selection of the optical elements, the aberration contributions due to each of the individual components can serve to cancel each other, thereby providing an aberration free system. For many of these designs, however, a sacrifice in the lens working distance must be made. Since advantages were provided by each of these approaches, both aspheric and multi-element designs were considered for this study.

A schematic representation of the layout used in design of the optical elements is given in Fig. 2. For this design, we assumed that the beam would fill the clear aperture of the input element. The input element radius was $25.4 \mathrm{~mm}$ and the clear aperture $r$ was defined as $80 \%$ of this radius. We then used the clear aperture radius and the measured beam half angle divergence $\theta$ to calculate the location of a point source which would fill the aperture. For design of these optical elements, the laser was assumed to be a point source located at a position $d$ away from the input lens face. The distance of the point source from the input element was taken to be

$$
d=\frac{r}{\tan \theta}
$$

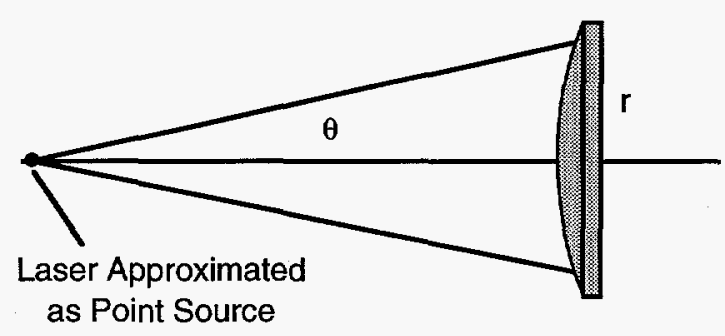

Figure 2. Schematic representation of configuration used to define boundary conditions for lens system design.

A commercially available lens design package was used to perform the optimization for each different design. Several iterations were required to arrive at a final design for each lens configuration. The first lens considered was a multi-element lens that would simply correct for spherical aberration and provide laser limited performance. This lens design is shown in Fig. 3. This lens consists of three elements and has an $\mathrm{f} / \# 1.14$. This design is fitted with a lens protector plate and provides a final working distance of $26.4 \mathrm{~mm}$. For this design, all spherical surfaces were used and each surface was anti-reflection coated to avoid heating of the lens housing due to reflection of the laser beam from each of the optical surfaces.

A second design considered was a singlet design that had an aspheric surface to correct for spherical aberration. The second surface was spherical. A schematic drawing of this lens design is given in Fig. 4. This design had an f/\# of 1 and a slightly longer working distance of $40 \mathrm{~mm}$. To produce the aspheric surface, hand grinding and polishing techniques were used. The cost was similar to that for the multi-element system. Again, fused silica was the lens material and all surfaces were anti-reflection coated. 
Based on the results of characterization of the first two lens designs, a third lens system was designed. This lens design, shown in Fig. 5, consisted of five elements and the emphasis of this design was to achieve a more functional system. Although the performance of the earlier multi-element lens design was very good, the limited working distance provided by this system was undesirable for welding application. The design chosen for the third and final design was a lens system referred to as an inverse-telephoto lens. The first three elements of this lens system serve to expand and collimate the laser beam, while the final two elements focus the expanded beam onto the sample surface. This design provided a numerical aperture slightly smaller than the two previous designs with a factor of two improvement in the working distance. It should be noted that the larger numerical aperture was due to design choices and was not inherent to the technique.

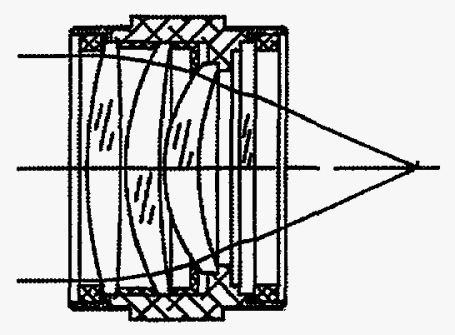

Figure 3. Layout of multi-element lens system design for initial performance testing.

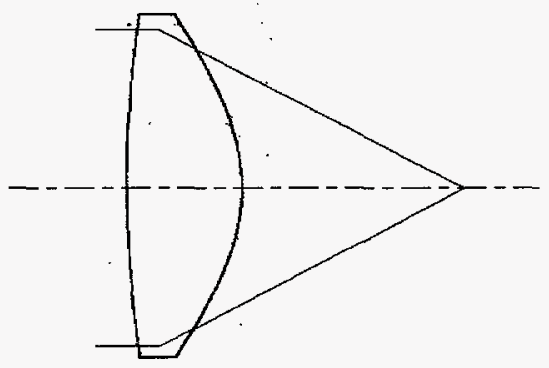

Figure 4. Layout of aspheric singlet lens design for initial performance testing.

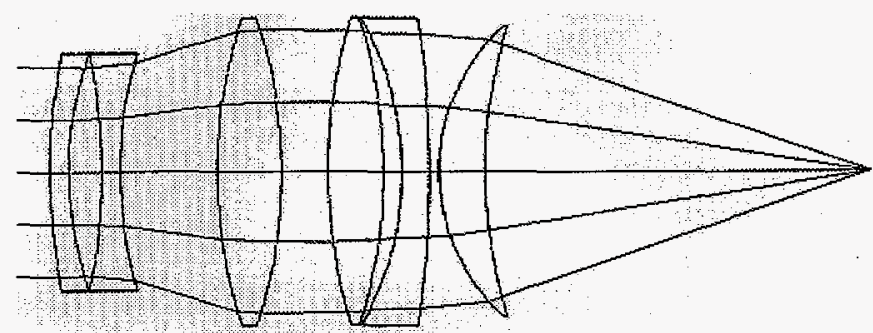

Figure 5. Layout of inverse-telephoto lens design for improved irradiance with a large working distance. 


\section{Characterization and Results}

Each of the lens designs was tested on the high power Nd:YAG laser to evaluate their performance. A commercially available beam diagnostic system was used to measure the focused beam radius at several positions along the beam optical axis through the minimum waist location. These measurements were performed for a series of power levels over the operating range of the laser. Measurements were made at two different times. The first set of measurements were taken to evaluate the performance of the $50.8 \mathrm{~mm}$ focal length multi-element and aspheric lens designs as compared to a $50.8 \mathrm{~mm}$ focal length plano-convex lens. Measurements from three different focal length plano-convex lenses, including a short focal length lens, were made to allow a theoretical focus curve to be established. The minimum focus spot size was selected as a measure of goodness of each lens design since it is, in general, the laser irradiance that limits the performance of a laser welding system ${ }^{[8]}$.

The measured spot size values versus lens focal length for each of the lens systems measured in the first set of tests is shown in Fig. 6. The long focal length plano-convex lens provided a data point where spherical aberration was known to be insignificant. A solid line is drawn through this point along a curve describing the predicted spot size as a function of focal length for an aberration free lens system. The multi-element lens design provides the best performance of the three short focal length lenses. The aspheric lens also provides improved performance as compared to the short focal length plano convex lens but there is still some aberration present which limits the performance of the aspheric lens design. Since both the aspheric and multi-element lenses were designed to provide diffraction limited performance, it is assumed that the fabrication technique used to produce the aspheric lens played a role in the performance deficiency. This is not to say that we could not have achieved diffraction limited performance from an aspheric lens but the cost would have been significantly higher than that for the multi-element lens system.

For the second set of tests, the performance of the $50.8 \mathrm{~mm}$ focal length multi-element lens was compared to that of the inverse-telephoto lens system. Again, spot size measurements were made at a series of positions along the focused beam optical axis through the minimum waist location. Since each of these lens systems had a different effective $f / \#$, the quality of the lenses was evaluated by comparing the ratio of the lens $\mathrm{f} / \#$ compared to the minimum waist spot size $w$. This ratio is given as

$$
\frac{f / \#_{\text {lens } 1}}{f / \#_{\text {lens } 2}}=\frac{w_{\text {lens } 1}}{w_{\text {lens } 2}} .
$$

The effective $f / \#$ values for the two lenses and the measured spot size values measured at a laser output power of $500 \mathrm{~W}$ are given in Table 1. It can be seen that the value for the ratio of the lens $\mathrm{f} / \#$ 's of 0.683 is essentially identical to the ratio value of 0.692 for the measured minimum 
waist values. This result suggests that the performance of each of these lens systems is equivalent. Since these lens systems provide equivalent performance, the graphical results (Fig. 6 ) for the multi-element lens in Fig. 3, suggesting near diffraction limited characteristics, would then imply similar near diffraction limited performance for the inverse-telephoto lens system.

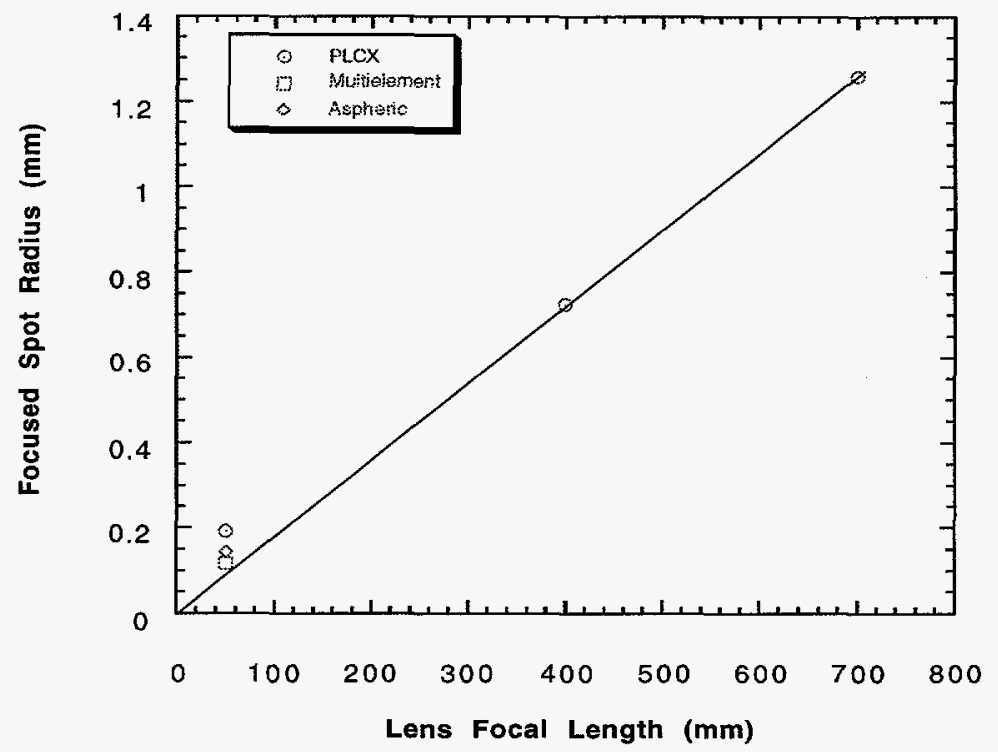

Figure 6. Measured focused laser spot size vs. lens focal length $(1320 \mathrm{~W})$.

Table 1. Lens parameters used to compare the performance of the inverse-telephoto lens system to the multi-element lens system ( $500 \mathrm{~W}$ laser power).

\begin{tabular}{|l|l|l|}
\hline Lens Design & Lens Effective f/\# & Measured Minimum Waist Radius $(\mu \mathrm{m})$ \\
\hline Multi-Element & 1.14 & 90 \\
\hline Inverse-Telephoto & 1.67 & 130 \\
\hline
\end{tabular}

Schematic representations of the multi-element and inverse telephoto lens designs are shown in Fig. 7. These drawings are shown using the same scale to emphasis the difference in the working distance between the two lens designs. As can be seen, the inverse telephoto lens system has a working distance that is twice that for the multi-element system. Yet there is only a $50 \%$ increase in the focused spot size. Since both lenses provide near diffraction limited performance, this result suggests that the inverse-telephoto lens design does indeed provide a solution for achieving high irradiance values with a working distance which is practical for volatile applications.

To demonstrate the feasibility of using these lens system for welding applications, a series of welds on 6061 aluminum were made at varying powers and travel speeds using the multi- 
element lens design. A metallographic cross-sectional view of a weld produced at 1400 watts, at $12.7 \mathrm{~mm} / \mathrm{s}$ and at sharp focus is shown in Fig. 8. For these welding experiments, there was an obvious hissing sound audible during welding, indicative of good coupling.

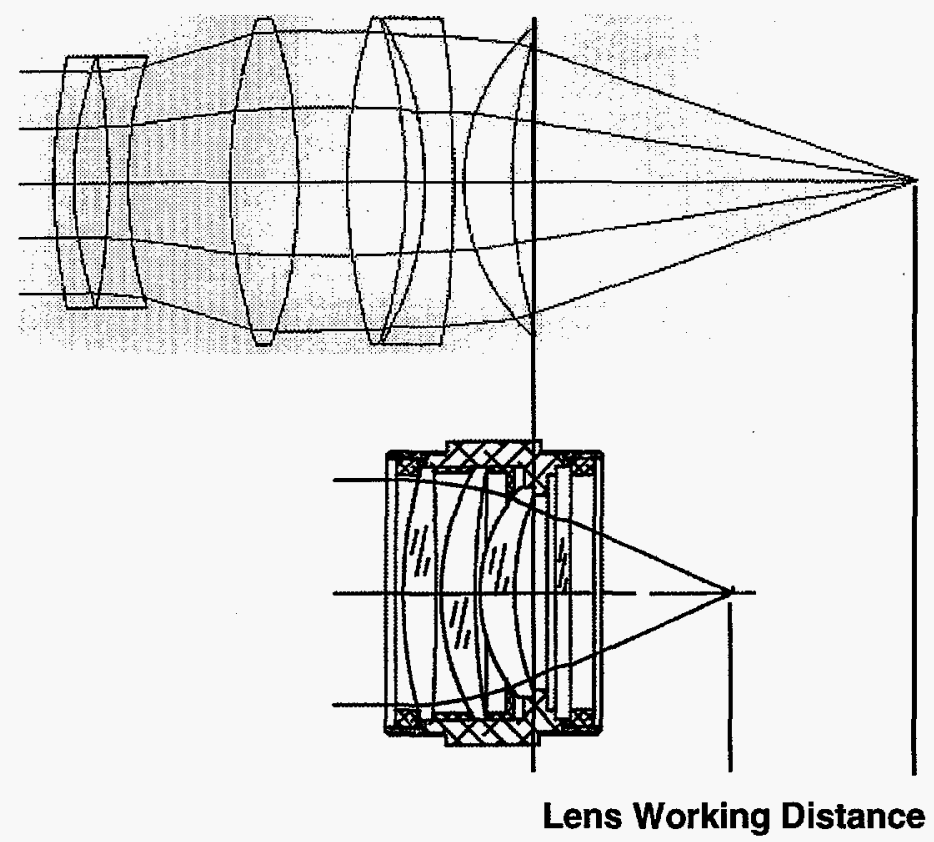

Figure 7. Scaled drawings of multi-element lens compared to inverse-telephoto lens.
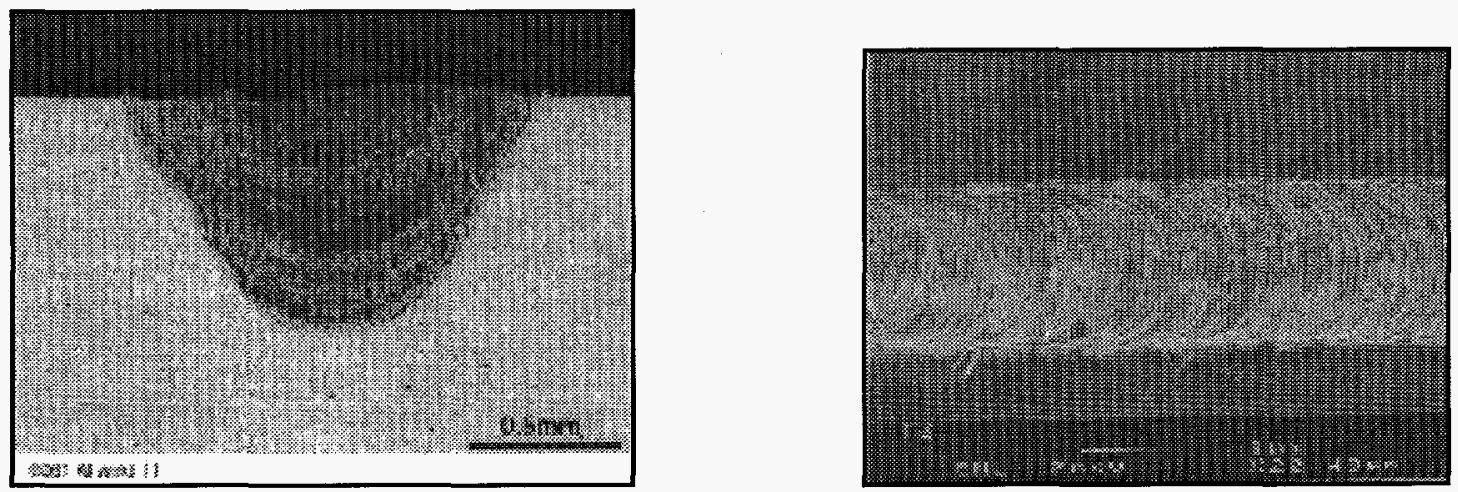

Figure 8. Laser weld on 6061 aluminum ( $1400 \mathrm{~W}, 12.7 \mathrm{~mm} / \mathrm{s})$.

\section{Conclusions}

Several lens systems were designed to overcome spherical aberration effects known to exist for high numerical aperture welding optics. The results of these experiments indicate that aspheric 
and multi-element lens systems can be used to accomplish this goal. However, the multi-element system provided the best results for this situation. Comparison of an inverse-telephoto lens to the multi-element lens design suggests similar near diffraction limited performance from both lens systems, yet the inverse-telephoto lens system provides a method to achieve a high irradiance with a working distance compatible with laser welding applications. The results of welding experiments demonstrate the feasibility of using these lens designs to achieve good weld penetration in 6061 aluminum.

\title{
Acknowledgments
}

The authors would like to thank Darrel Frear for his insightful input and review of this manuscript.

* This work was performed at Sandia National Laboratories supported by the U. S. Department of Energy under contract number DE-AC04-94AL85000.

\section{$\underline{\text { References }}$}

1. I. W. Boyd, Laser Processing of Thin Films and Microstructures, Springer-Verlag, Berlin, 1987, pg. 29.

2. T. Ishide, O. Matsumoto, Y. Nagura and T. Nagashima, "Optical fiber transmission of 2 kW CW YAG laser and its practical application to welding", SPIE v 1277, 188-198, (1990).

3. M. W. Sasnett and T. F. Johnston, Jr., "Beam characterization and measurement of propagation attributes", SPIE Vol. 1414 Laser Beam Diagnostics, 21-32 (1991).

4. D. M. Keicher, "Laser Beam Characterization Results for a High Power CW Nd:YAG Laser”, SPIE v. 2375, 162-171, (1995).

5. M. Essien and P. W. Fuerschbach, "Beam Characterization of a Materials Processing $\mathrm{CO}_{2}$ Laser", submitted to Welding Journal Jan., 93.

6. M. Essien, L. P. Schanwald and P. W. Fuerschbach, "Spotsize measurements of a CW Nd:YAG laser", SPIE v. 2375, 156-161, (1995).

7. M. V. Klein and T. E. Furtak, Optics, John Wiley \& Sons, 1986.

8. P. W. Fuerschbach, "Measurement and Prediction of Energy Transfer Efficiency in Laser Beam Welding", submitted to Welding Journal.

\section{DISCLAIMER}

\begin{abstract}
This report was prepared as an account of work sponsored by an agency of the United States Government. Neither the United States Government nor any agency thereof, nor any of their employees, makes any warranty, express or implied, or assumes any legal liability or responsibility for the accuracy, completeness, or usefulness of any information, apparatus, product, or process disclosed, or represents that its use would not infringe privately owned rights. Reference herein to any specific commercial product, process, or service by trade name, trademark, manufacturer, or otherwise does not necessarily constitute or imply its endorsement, recommendation, or favoring by the United States Government or any agency thereof. The views and opinions of authors expressed herein do not necessarily state or reflect those of the United States Government or any agency thereof.
\end{abstract}

